\title{
An Effective Strategy for the Synthesis of Biocompatible Gold Nanoparticles Using Cinnamon Phytochemicals for Phantom CT Imaging and Photoacoustic Detection of Cancerous Cells
}

Nripen Chanda, ${ }^{1,5}$ Ravi Shukla, ${ }^{1}$ Ajit Zambre, ${ }^{1}$ Swapna Mekapothula, ${ }^{1}$ Rajesh R. Kulkarni, ${ }^{1}$ Kavita Katti, ${ }^{1}$ Kiran Bhattacharyya, ${ }^{2}$ Genevieve M. Fent, ${ }^{3}$ Stan W. Casteel, ${ }^{3}$ Evan J. Boote, ${ }^{1}$ John A. Viator, ${ }^{2}$ Anandhi Upendran, ${ }^{4}$ Raghuraman Kannan, ${ }^{1,5}$ Kattesh V. Katti ${ }^{1,5}$

${ }^{1}$ Department of Radiology, 301 Business Loop 70W, University of Missouri, Columbia, Missouri, USA

${ }^{2}$ Department of Biological Engineering, University of Missouri, Columbia, Missouri, USA

${ }^{3}$ Department of Veterinary Pathobiology, University of Missouri, Columbia, Missouri, USA

${ }^{4}$ Nanoparticle Biochem, Inc., Columbia, MO 65201, USA

${ }^{5}$ Correspondence Email Addresses: chandan@missouri.edu, kattik@ health.missouri.edu, kannanr@health.missouri.edu

Running Head: Cinnamon Gold Nanoparticles for Imaging and Detection

Key Words: Cinnamon stabilized gold nanoparticles, cellular internalization, cancer cells, phantom CT imaging, photoacoustic detection and in vivo biodistribution. 


\section{ABSTRACT}

Purpose. The purpose of the present study was to explore the utilization of cinnamon coated gold nanoparticles (Cin-AuNPs) as CT/optical contrast enhancement agent for detection of cancer cells.

Methods. Cin-AuNPs were synthesized by a "Green" procedure and the detailed characterization has been performed by physic-chemical analysis. Cytotoxicity and cellualar uptake studies were carried out in normal human fibroblast and cancerous (PC-3 and MCF-7) cells respectively. The efficacy of detecting cancerous cells was monitored using photoacoustic technique. In vivo biodistribution was studied after IV injection of Cin-AuNPs in mice and a CT phantom model was generated.

Results. Biocompatible Cin-AuNPs were synthesized with high purity. Significant uptake of these gold nanoparticles was observed in PC-3 and MCF-7 cells. Cin-AuNPs internalized in cancerous cells facilitate detectable photoacoustic signals. In vivo biodistribution in normal mouse shows steady accumulation of gold nanoparticles in lungs and rapid clearance from blood. Quantitative analysis of CT values in phantom model reveals that the cinnamon phytochemicals coated AuNPs has reasonable attenuation efficiency.

Conclusions. The results indicate that these non-toxic Cin-AuNPs can serve as excellent CT/ photoacoustic contrast enhancement agents and may provide a novel approach toward the tumor detection through nanopharmaceuticals. 


\section{INTRODUCTION}

Nanotechnology is poised for rapid growth with myriad applications in pharmaceutical research, smart electronic materials, alternative energy generation, environmental restoration, and allied fields.(1-4) Development of large-scale production technologies for both metallic and non-metallic nanoparticles with little or no collateral environmental pollution is becoming a basic necessity, with significant scientific and societal relevance.

Toxic chemicals are utilized in several of the processes for production of nanoparticles, either in the form of reducing agents to reduce various metal salts to their corresponding nanoparticles, or as stabilizing agents to prevent agglomeration of nanoparticles. For example, hydrazine and sodium borohydride are powerful reducing agents that are currently used to produce gold and other metallic nanoparticles. $(5,6)$ These reducing agents are highly toxic to living organisms and to the environment, and due care must be exercised in their proper handling and disposal of toxic chemicals.

Our results indicate that large scale production of nanoparticles using phytochemicals occurring in natural resources such as plant species will minimize or eliminate chemical interventions, thereby resulting in true green and non-polluting ecofriendly industrial processes for the manufacture of nanoparticle-based smart materials.(7-14)

Various herbs and plant sources occlude powerful antioxidants that are present as photochemical constituents in seeds, stems, fruits and in leaves. $(15,16)$ These naturally occurring antioxidants have existed in human food chain for thousands of years, and are known to be non-toxic to living organisms and to the environment. $(17,18)$ The utility of 
plant based phytochemicals in the overall synthesis and architecture of nanoparticles and various nanoparticle embedded products creates an important symbiosis between natural/plant sciences and nanotechnology. This connection provides an inherently green approach to nanotechnology, referred to as "green nanotechnology". We have recently reported the application of phytochemicals available within soy, tea and cumin as dual reducing and stabilizing agents for the synthesis of gold nanoparticles. $(8,12-14)$ We herein report the utility of phytochemicals occluded within cinnamon as reducing and stabilizing agents to synthesize biocompatible gold nanoparticles from the precursor gold salts. Phytochemical constituents of cinnamon include up to 1-4\% of an essential oil, 510\% polyphenols, $80-90 \%$ carbohydrates, and other ingredients that include gum, mucilage, resin and calcium monoterpenes oxalate.(19-24) The essential oil has been characterized as primarily aldehydes (up to 60-80\%) including trans-cinnamaldehyde. The primary phytochemicals that provide the characteristic aroma of cinnamon is cinnamaldehyde in combination with other related organic alcohols.(20-24)

The phytoconstituents embedded within cinnamon contain functional groups such as aldehyde and hydroxyl units within the molecular framework, in combination with carbohydrates (starch and polysaccharides). We hypothesize that these functional groups will provide synergistic chemical reduction power for the production and stabilization of gold nanoparticles in a singular green process. The rationale behind this hypothesis is based on the reduction capabilities of a cocktail of phytochemicals present in cinnamon and their ability to chemically reduce gold (III) salts to nanoparticles with consequent coating of carbohydrates, as well as a host of other phytochemicals present in cinnamon that can stabilize the freshly generated gold nanoparticles. The validation of this 
hypothesis will result in a versatile 'Green Nanotechnology,' with subsequent production of gold nanoparticles for the use in a variety of applications in nanomedicine and pharmaceutical arena. Although significant research has been made in the production of phytochemical coated gold nano-constructs, little is known about their biodistribution and toxicity under in vivo condition.(8-9,12-14) In fact, guidelines for evaluating the efficacy and toxicity of these nanoparticles for human use are still in the evolutionary phase. In this scenario, it is prudent to investigate and understand the nature of phytochemical stabilized nanoparticles, under in vivo conditions, in ways adopted for the development of various pharmaceuticals.

In this paper, we describe detailed investigations encompassing (i) cinnamon phytochemicals mediated synthesis of gold nanoparticles (Cin-AuNPs); detailed characterization including size and shape analysis; (ii) cellular toxicity (cytotoxicity) on normal human fibroblast cells; (iii) cellular internalization efficacy of Cin-AuNPs under in vitro conditions with cancerous (PC-3 and MCF-7) cells; (iv) efficacy of Cin-AuNPs toward in vitro detection of cancerous cells (PC-3) using photoacoustic technique; (v) in vivo biodistribution of Cin-AuNPs in normal mice; and (iv) ability of Cin-AuNPs to serve as contrast enhancers for use in $\mathrm{CT}$ molecular imaging. 


\section{MATERIALS AND METHOD}

\section{Materials}

The components (natural constructs) used in the synthesis of gold nanoparticles (AuNPs) were procured from standard vendors. $\mathrm{NaAuCl}_{4}$ was purchased from AlfaAesar. Trans-cinnamaldehyde, trans-cinnamic acid, eugenol, cinnamyl alcohol, cinnamyl acetatel, linalool, $\alpha$-terpinene, R-(+)-Limonene, eugenyl acetate, linalyl acetate, catechin, and epicatechin were purchased from Sigma (St. Louis, MO, USA). All cell culture materials e.g. minimum essential medium (MEM with nonessential amino acids, powdered), HEPES, bovine insulin, streptomycin sulfate, and penicillin-G, were obtained from Sigma (St. Louis, MO, USA). Bovine calf serum, phenol red (sodium salt), and lyophilized trypsin were obtained from Gibco BRL (Grand Island, NY). Human fibroblasts primary cultures were obtained from Prof. Cris Lorson, Bond Life Science Center, (University of Missouri, Columbia, MO). Fibroblast cells were maintained in

DMEM with $10 \mathrm{pgmL}^{-1}$ phenol red, $10 \mathrm{mM}$ HEPES, 100 units $\mathrm{mL}^{-1}$ penicillin, 100 $\operatorname{pgmL}^{-1}$ streptomycin, and $10 \%$ donor bovine serum (maintenance medium).

\section{Measurements}

Transmission Electron Microscope (TEM) images were obtained on a JEOL 1400 TEM (JEOL, LTE, Tokyo, Japan). The absorption measurements were done using a Varian Cary 50 UV-Vis spectrophotometer and disposable cuvettes with a volume of 1 $\mathrm{mL}$ and a path length of $10 \mathrm{~mm}$. The core size of the nanoparticles was measured on DC 24000, CPS Instruments Inc. USA. The hydrodynamic diameter and zeta potential were obtained using Zetasizer Nano S90 (Malvern Instruments Ltd. USA).

\section{Synthesis of Cinnamon Stabilized Gold Nanoparticles (Cin-AuNPs)}


To a $20 \mathrm{~mL}$ vial was added $6 \mathrm{~mL}$ of doubly deionized (DI) water followed by the addition of $25 \mathrm{mg}$ of cinnamon. The mixture was stirred continuously at $25^{\circ} \mathrm{C}$ for $5 \mathrm{~min}$. To the stirring mixture was added $100 \mu \mathrm{L}$ of $0.1 \mathrm{M} \mathrm{NaAuCl}_{4}$ solution (in DI water). The color of the mixture turned purple within 20 minutes after the addition of $\mathrm{NaAuCl}_{4}$ indicating the formation of gold nanoparticles. The reaction mixture was stirred additionally for 10 minutes. The gold nanoparticle solution was separated from the residual cinnamon powder by means of a standard paper filter. The solution was further filtered through a 0.5 micron filter. The gold nanopaticles were characterized by UV-Vis absorption spectroscopy and Transmission Electron Microscopy (TEM) analysis.

\section{Generation of Gold Nanoparticles (AuNPs) by Individual Cinnamon Phyto- constituents}

Attempted synthesis of gold nanoparticles from $\mathrm{NaAuCl}_{4}$ has been done with each of the major components present in cinnamon as phytochemicals. All the alcoholic components present in cinnamon produce gold nanoparticles by reducing $\mathrm{NaAuCl}_{4}$, but fail to stabilize in aqueous medium. To provide stabilization, starch (present as carbohydrate (80-90\%) in cinnamon) was used for the gold nanoparticles production process. The procedure for making gold nanoparticles by cinnamon components (linalool, epicatechin, catechin and trans-cinnamaldehyde) is as follows: To a $20 \mathrm{~mL}$ vial was added $6 \mathrm{~mL}$ of doubly deionized (DI) water followed by the addition of $22.5 \mathrm{mg}$ of starch. The mixture was stirred continuously at $\sim 80^{\circ} \mathrm{C}$ for $10 \mathrm{~min}$. After dissolving the starch, the solution was cooled down to $\sim 30^{\circ} \mathrm{C}$ with continuous stirring. To this solution was added $10 \mathrm{mg}$ of linalool or $2.5 \mathrm{mg}$ catechin or $2.5 \mathrm{mg}$ epicatechin followed by 100 $\mu \mathrm{L}$ of $0.1 \mathrm{M} \mathrm{NaAuCl}_{4}$ solution (in DI water). The color of the mixture turned purple-red 
within 30 minutes after the addition of $\mathrm{NaAuCl}_{4}$ indicating the formation of gold nanoparticles. The addition of starch is not required for the synthesis of transcinnamaldehyde-AuNPs. The purple-red solutions were characterized by UV-Vis absorption spectroscopy and TEM analysis.

\section{In vitro Stability Studies}

In vitro stability studies of Cin-AuNPs were performed by mixing gold nanoparticles to aqueous solutions of $10 \% \mathrm{NaCl}, 0.5 \%$ cysteine, $0.2 \mathrm{M}$ histidine, $0.5 \%$ HSA and $0.5 \%$ BSA. The stability of the conjugates was measured by monitoring the UV absorbance over a period of $2 \mathrm{hrs}, 24 \mathrm{hrs}$ and 7 days. A negligible change in UV-vis plasmon band confirmed the retention of nanoparticulate composition in all mixtures. TEM studies further confirmed the stability of the nanoparticles in all biologically relevant media.

\section{In vitro Cytotoxicity Measurements (MTT Assay)}

In vitro cytotoxicity evaluation of Cin-AuNPs was performed as described by the supplier (ATCC, USA). Briefly, $2 \times 10^{4}$ normal fibroblasts cells at the exponential growth phase were seeded in each well of a flat-bottomed 96-well polystyrene-coated plate and were incubated at $37{ }^{\circ} \mathrm{C}$ for $24 \mathrm{~h}$ in $\mathrm{CO}_{2}$ incubator at $5 \% \mathrm{CO}_{2}$ environment. Series of concentrations such as $0,0.25,0.5,0.75$ and $1 \mathrm{mg} / \mathrm{mL}$ of these nanoparticles were made in the medium. Each concentration was added to the plate in pentaplet manner. After $24 \mathrm{~h}$ incubation, $10 \mu \mathrm{L}$ per well MTT (stock solution $5 \mathrm{mgmL}^{-1} \mathrm{PBS}$ ) (ATCC, USA) was added and kept for $24 \mathrm{~h}$ and the formosan crystals so formed were dissolved in $100 \mu \mathrm{L}$ detergent. The plates were kept for $18 \mathrm{~h}$ in dark at $25^{\circ} \mathrm{C}$ to dissolve all crystals, and the intensity of developed color was measured by micro plate reader 
(Dynastic MR 5000, USA) operating at $570 \mathrm{~nm}$ wavelength. Wells with complete medium, nanoparticles, and MTT-but without cells-were used as blanks. Untreated cells were considered $100 \%$ viable.

\section{Cellular Uptake Studies}

Breast cancer (MCF-7) and prostate cancer (PC-3) cells obtained from ATCC were used for in vitro cell internalization analysis. MCF-7 cells were maintained in MEM with nonessential amino acids, $10 \mathrm{pg} / \mathrm{ml}$ phenol red, $10 \mathrm{mM}$ HEPES, $6 \mathrm{ng} / \mathrm{ml}$ insulin, 100 units/ml penicillin, $100 \mathrm{pg} / \mathrm{ml}$ streptomycin, and 10\% FBS (maintenance medium). PC-3 cells were maintained in RPMI medium supplemented with $4.5 \mathrm{~g} / \mathrm{L}$ D-glucose, $25 \mathrm{mM}$ HEPES,0.11 g/L sodium pyruvate, $1.5 \mathrm{~g} / \mathrm{L}$ sodium bi carbonate, $2 \mathrm{mM}$ L-glutamine and $10 \%$ FBS and antibiotics. Known concentrations of Cin-AuNPs $(100 \mu \mathrm{g} / \mathrm{mL})$ were added to PC-3 and MCF-7 cells ( 10000 cells) and Cinnamaldehyde-AuNPs $(100 \mu \mathrm{g} / \mathrm{mL})$ to only PC-3 cells followed by 4 hours of incubation at $37^{\circ} \mathrm{C}$. The cells were washed three times with PBS, centrifuged into small pellets, and fixed with $2 \%$ glutaraldehyde $2 \%$ paraformaldehyde in sodium cacodylate buffer $(0.1 \mathrm{M})$. The cells were further fixed with $1 \%$ buffered osmium tetraoxide and dehydrated in an ethanol series before embedding in Epon-Spurr epoxy resin. Sections $(75-85 \mathrm{~nm})$ were cut using a Leica Ultracut UCT ultramicrotome and placed on a TEM grid. The sections were post-stained with uranyl acetate and lead citrate for organelle visualization. The prepared samples were viewed with a JEOL 1400 TEM.

\section{Prostate Cancer Cell Imaging Using Photoacoustic Technique}

Prostate cancer cells (PC-3) were maintained in RPMI medium supplemented with $4.5 \mathrm{~g} / \mathrm{L}$ D-glucose, $25 \mathrm{mM}$ HEPES, $0.11 \mathrm{~g} / \mathrm{L}$ sodium pyruvate, $1.5 \mathrm{~g} / \mathrm{L}$ sodium 
bicarbonate, $2 \mathrm{mM} \mathrm{L-glutamine} \mathrm{and} 10 \%$ FBS and antibiotics. Actively growing cells were seeded in $60 \mathrm{~mm}$ tissue culture dishes at a cell seeding density of $1.0 \times 10^{5}$ cells $/ \mathrm{ml}$ and were incubated for 48 hours in a $\mathrm{CO}_{2}$ incubator at $37^{\circ} \mathrm{C}$. The cells were washed two times with isothermal medium followed by treatment with $100 \mu \mathrm{g} / \mathrm{ml}$ Cin-AuNPs for 24 hours. In untreated control cells, an equal volume of sterile milli $\mathrm{Q}$ water was added. At the end of incubation, the cells were washed five times with plain RPMI medium followed by ten times with phosphate buffered saline to remove any free gold. The cells were dislodged by an enzymatic method and enzyme traces were removed by centrifugation and resuspension of the cell pellet in PBS. Finally, the cells were fixed in paraformaldehyde solution and were utilized for photoacoustic detection. A frequency tripled Nd:YAG laser pumped optical parametric oscillator that was tunable from 410$2400 \mathrm{~nm}$ (Vibrant II, Opotek, Carlsbad, California) was used as the excitation source. The pulse energy was approximately $5 \mathrm{~mJ}$. The exact pulse energy was monitored using an energy meter (Field Max, Coherent, Beaverton, Oregon). The laser pulse duration was $5 \mathrm{~ns}$ and coupled into a 1000 micron optical fiber. The setup was performed using optical reflectance at an interface in the manner of Paltauf et al. (25) This method relies on index of refraction changes in the liquid medium within which photoacoustic waves propagate, thereby changing the reflectance of a laser probe beam. This method is preferred for circulating tumor cells (CTC) detection as it is immune to pyroelectric effects caused by high laser fluence impinging upon polymer sensing films. Using this system, Cin-AuNPs tagged PC-3 cells were suspended in normal saline in a microcuvette. The active area of the sensor was the laser beam volume in the microcuvette which was approximately 1 micrometer. A concentration of $3.5 \times 10^{5}$ cells/milliliter resulted in 
irradiation of approximately 350 cells. These measurements were repeated five times for five different concentrations $\left(3.5 \times 10^{5}, 1 \times 10^{5}, 5 \times 10^{4}, 1.5 \times 10^{4}\right.$, and $1.0 \times 10^{4}$ cells/ml). The Signal to Noise Ratio (SNR) for the lower concentrations was similar due to difficulties in precise estimation of cell number and the variability of nanoparticle content in each cell.

\section{Biodistribution of Cin-AuNPs in Mice Model}

The biodistribution of Cin-AuNPs were assessed in two groups of mice ( $\mathrm{n}=3$ ) with each mouse weighing $\sim 20 \mathrm{~g}$. The measurements were done by estimating the amount of gold in various organs using fAAS. For each time point, animals were intravenously injected with Cin-AuNPs $(200 \mu \mathrm{L}, 3.75 \mathrm{mg} / \mathrm{mL})$ through tail and sacrificed after $4 \mathrm{~h}$ and $24 \mathrm{~h}$ time periods. The amount of gold in blood, brain, heart, kidney, pancreas, tumor, spleen, lung and liver were determined to assess the tissue affinity for Cin-AuNPs.

Sample Preparation for fAAS: Tissues were removed from $-80^{\circ} \mathrm{C}$ storage and allowed to partially thaw. Following partial thawing, whole tissue samples were placed in a $15 \mathrm{ml}$ clean graduated centrifuge tube with concentrated trace metal grade nitric acid (Fisher Scientific) and concentrated trace metal grade hydrochloric acid in 1:2 ratio and heated in an oven to $85^{\circ} \mathrm{C}$ overnight $(12-18 \mathrm{hr})$. The amount of acid depended on the weight of the tissue and the proportion used was $1 \mathrm{mg}$ (tissue): $1 \mu \mathrm{L}\left(\mathrm{HNO}_{3}\right): 0.5 \mu \mathrm{L}$ $(\mathrm{HCl})$. After cooling, the digest was diluted in 1:10 ratio with ultra pure water for analysis. The mouse carcasses were homogenized by grinding into a fine powder using a stein mill and then $\sim 1 \mathrm{~g}$ aliquots were digested and analyzed with the method described for the other mouse tissues. 
fAAS Analysis: All the samples were analyzed by furnace AAS using a standard curve spanning $0-100$ micrograms $/ \mathrm{L}$. The furnace parameters were as specified in the user's manual for the Perkin-Elmer Analyst 800 ThGA graphite furnace. Quality-control materials (duplicates, spikes, and instrument-calibration verification) were within appropriate ranges.

\section{CT Imaging}

Samples containing solutions of Cin-AuNPs were scanned using a Siemens Definium CT system. Five 5.0-mL glass vials containing aqueous solutions of various concentrations of Cin-AuNPs were placed in holders alongside six similar vials filled with distilled water. A simultaneous dual-energy scan was acquired at tube voltages of 80 and $140 \mathrm{kVp}$. Images were reconstructed in $0.6 \mathrm{~mm}$-thick slices with a field of view of $120 \mathrm{~mm}$. Evaluation of the contrast enhancement contribution of Cin-AuNPs was carried out by loading the digital CT images in a standard display program and then selecting a region of interest on the resultant CT image for each sample and the background.(26) Contrast enhancement was determined in $\Delta \mathrm{HU}$ for each mass concentration of Cin-AuNPs and each tube voltage.

\section{RESULTS}

\section{Synthesis and Characterization of Cinnamon-Mediated Gold Nanoparticles}

Cinnamon phytochemicals stabilized gold nanoparticles (Cin-AuNPs) were prepared by simple mixing an aqueous solution of commercially available sodium tetrachloroaurate with cinnamon in DI water. The reaction produced a purple-red coloration within 25 minutes at room temperature. The absorbance profile of these 
nanoparticles is shown in Fig. 1. The surface plasmon resonance appeared at $\sim 540 \mathrm{~nm}$ $\left(\lambda_{\max }=540 \mathrm{~nm}\right)$. Transmission Electron Microscopy $(\mathrm{TEM})$ and Differential Centrifugal Sedimentation (DCS) techniques were used to determine the core size while Dynamic Light Scattering (DLS) was used to evaluate the hydrodynamic size of phytochemicals coated gold nanoparticles (Table 1). As seen in the TEM image (inset, Fig. 1), the size and shape of the nanoparticles are homogeneous with an average diameter of $13 \pm 5 \mathrm{~nm}$, depicting a narrow size distribution. The hydrodynamic size $(155 \mathrm{~nm})$ of cinnamon coated gold nanopaprticles is greater than the core size measured by TEM and DCS $(32 \pm 2 \mathrm{~nm})$ techniques. The negative zeta potential $(\zeta)$ value $(-31.0 \mathrm{mV})$ for the CinAuNPs provide the necessary repulsive forces for the particles to remain stable in solution. In general, zeta potential provides pivotal information on the dispersion of nanoparticles as the magnitude of the charge is an indication of the mutual repulsion between particles that can be used to predict the long-term stability of the nanoparticulate dispersion.

Gold nanoparticles using commercially available individual constituents of cinnamon were synthesized to determine the critical roles of the various phytochemicals present in cinnamon. Results of these experiments have unambiguously confirmed that cinnamaldehyde and linalool are the primary reducing agents to reduce $\mathrm{Au}(\mathrm{III})$ to gold nanoparticles. The carbohydrates present in cinnamon act as stabilizers for the gold nanoparticles. The progress of all the individual reactions was monitored by UV visible absorption spectroscopy and the representative spectra of cinnamaldehyde and linalool produced gold nanoparticles are shown in Fig. 2.

\section{In vitro Stability Studies}


Biomedical imaging application requires stability of Cin-AuNPs over a reasonable length of time. $(8,10,12,13)$ In vitro stability tests were performed by incubating solutions of Cin-AuNPs with $10 \% \mathrm{NaCl}$, cysteine, histidine, Human Serum Albumin (HSA) and Bovine Serum Albumin (BSA) solutions that mimic biological environments. The stability and the identity of Cin-AuNPs were measured by recording UV absorbance after 24 hours, as shown in Fig. 3. The plasmon resonance band at $~ 535$ $\mathrm{nm}$ confirmed the retention of nanoparticulates in all the above mixtures. This retention indicates that the AuNPs are intact, and thereby demonstrate excellent in vitro stability in biological fluids at physiological $\mathrm{pH}$. TEM and zeta potential measurements also confirmed the retention of the nanoparticulate compositions in all of the above media, thus signifying the robust nature of these nanoparticles under in vitro conditions. CinAuNPs demonstrated excellent in vitro stability under $\mathrm{pH} 4$ to 9 range implying that these nanoparticles can be used in a wide $\mathrm{pH}$ range for various biomedical applications.

\section{In vitro Cytotoxicity Measurements}

Although the stability of Cin-AuNPs is excellent in in vitro, it is important to check their biocompatibility for in vivo applications. $(8,10,12,13)$ The cytotoxicity of Cin-AuNPs was studied on primary human fibroblast cells under in vitro conditions using a colorimetric cell-viability (MTT) assay, as shown in Fig. 4. In MTT assay, the cell viability was examined by the absorbance of formazan (produced by the cleavage of MTT by dehydrogenases in living cells) at $570 \mathrm{~nm}$ which is directly proportional to the number of live cells. The experiment was performed using a wide range of concentrations of Cin-AuNPs $(0,0.25,0.5,0.75$ and $1 \mathrm{mg} / \mathrm{mL})$. The relative cell viability was $\sim 90 \%$ for both $24 \mathrm{~h}$ post treatment, not significantly different from the control. 


\section{Cellular Internalization}

Gold nanoparticles are currently investigated for their potential applications in drug delivery, therapy and as intracellular molecular imaging applications.(27-33) Cellular internalization studies of Cin-AuNPs will provide insights into our understanding of the scope of using these nanoparticles in site-specific and cell-specific delivery. A number of studies have demonstrated that phytochemical-coated gold nanoparticles have the ability to penetrate cell membranes and internalize within the cellular matrix. $(8,12,34,35)$ Accordingly, the internalization propensity of cinnamon phytochemicals coated gold nanoparticles in MCF-7 and PC-3 cancer cells of human origin were tested. After careful mixing of Cin-AuNPs to media of both cellular types, the gold nanoparticles incubated cells were washed vigorously using trypsin-EDTA solution followed by PBS ( $\mathrm{pH} 7.4$ ) to remove any unbound gold nanoparticles. Figure 5 shows the TEM images of Cin-AuNPs after their internalization within the cells. The results clearly demonstrate that Cin-AuNPs are internalized into both prostate as well as breast cancerous cells and the identity of individual nanoparticles appeared intact inside the cells.

\section{Cocktail Phytochemical Effects on Cellular Internalization}

Cinnamon occludes essential oils in which $60-80 \%$ is cinnamaldehyde. $(19,20,22$, 23, 36) Independent experiments were performed to produce gold nanoparticles upon interactions of sodium teterachloroaurate with cinnamaldehyde, a powerful reducing agent. In order to understand the relative effects of a cocktail of phytochemicals in cinnamon versus cinnamaldehyde on the overall propensity for cellular internalization of AuNPs, a comparison was made on the relative uptake of Cin-AuNPs with the AuNPs 
produced by cinnamaldehyde only. It is interesting to note that cinnamaldehyde synthesized AuNPs (cinnamaldehyde-AuNPs) showed significantly lower uptake of AuNPs in PC-3 cells as compared to the excellent propensity for cellular internalization as shown by Cin-AuNPs (Fig. 5). This observation strongly proves that the coating of a cocktail of phytochemicals present in cinnamon onto Cin-AuNPs plays an important role in internalization of AuNPs into cancer cells, and the coating of only cinnamaldehyde onto AuNPs results in limited or no internalization into prostate cancer cells.

\section{In-vitro Detection of AuNPs in Circulating Cancer Cells Using Photoacoustic}

\section{Imaging}

Gold nanoparticles embedded tumor cells offer significant advantages for photoacoustic detection of tumor cells.(37-41) As a proof of concept to identify circulating PC-3 cells, we have treated cells with Cin-AuNPs using established procedures.(41) The PA experiment was performed at five different concentrations, $3.5 \times 10^{5}, 1 \times 10^{5}, 5 \times 10^{4}, 1.5 \times 10^{4}$, and $1.0 \times 10^{4}$ cells $/ \mathrm{ml}$ which resulted in irradiation of approximately $350,100,50,15$ and 10 cells respectively. A comparison of the signals obtained for the untreated/treated Cin-AuNPs cells are shown in Figure 6. As a representative illustration, photoacoustic signal obtained by irradiation of 15 Cin-AuNPs tagged PC-3 cells is shown in Figure 6 (right). The untreated PC-3 cells upon irradiation, showed a linear photoacoustic response validating the efficacy of utilizing Cin-AuNPs to detect circulating prostate cancer cells.

\section{In vivo Biodistribution Studies}

In vivo biodistribution studies were performed in normal mice model to determine the pharmacokinetics of Cin-AuNPs. The Cin-AuNPs $(3.75 \mathrm{mg} / \mathrm{mL}$ of $\mathrm{Au})$ were injected 
intravenously in two groups of mice $(\mathrm{n}=3)$, each mouse weighing $\sim 20$ g. Following 200 $\mu \mathrm{L}(0.75 \mathrm{mg}$ of $\mathrm{Au})$ intravenous administrations of Cin-AuNPs post injection periods $4 \mathrm{~h}$ and 24hrs, the tissues and fluids in mice were harvested for estimation of gold by Atomic Absorption Spectroscopy (AAS). The results of biodistribution studies for the intravenous route of administration of Cin-AuNPs as estimated by AAS are depicted in

Fig. 7. The accumulation and clearance of AuNPs in various organs as shown in Fig. 7 demonstrate the limited binding with blood-plasma proteins, which also signifies the high in vivo stability, presumably due to the effective coating of the phytochemicals around the AuNPs. The significant accumulations of Cin-AuNPs ( $482.0 \mu \mathrm{g} / \mathrm{g}$ of tissue) in lung were almost the same from $4 \mathrm{~h}$ to $24 \mathrm{~h}$. In addition, there is also a gradual accumulation of gold nanoparticles in liver and spleen for up to $24 \mathrm{~h}$.

\section{X-Ray CT Contrast Enhancement:}

CT imaging analysis on phantoms was used to evaluate the ability of Cin-AuNPs as contrast enhancement agents. A simultaneous dual-energy scan was measured at 80 and $140 \mathrm{kVp}$ as shown in Fig. 8. The contrast differential $\Delta \mathrm{HU}$ (Hounsfield Unit) values were measured for increasing concentration of Cin-AuNPs (Fig. 9). The slopes of both lines $(80$ and $140 \mathrm{kVp}$ ) are approximately $18.5 \mathrm{HU}$ per milligram of gold per $\mathrm{mL}$ of volume in comparison to water.

\section{DISCUSSION}

Phytochemicals within cinnamon constitute essential oil (trans-cinnamaldehyde, eugenol, linalool, trans-cinnamic acid, terpenes and others; $1-4 \%$ by weight), polyphenols (catechin, epicatechin, anthocyanidin, catechin/epicatechin oligomers, kaempferitrin and 
others; $5-10 \%$ by weight) and carbohydrates (starch, polysaccharides, ash; $80-90 \%$ by weight, Table 2). These components contain active functional groups such as hydroxyl, aldehyde and carboxyl units which may play pivotal roles in providing synergistic chemical reduction power for the reduction of $\mathrm{NaAuCl}_{4}$ into AuNPs. Gold nanoparticles produced by using a cocktail of phytochemicals in cinnamon did not require any external chemicals to stabilize the nanoparticulate matrix. Various carbohydrates (starch, glucose, arabinose, galactose, rhamnose and combination thereof) present in cinnamon (Table 2) are presumably responsible for making a robust coating on gold nanoparticles and rendering stability against agglomerations.

In the present study, the roles of various phytochemicals for the generation and stabilization of AuNPs by interactions with $\mathrm{NaAuCl}_{4}$ in aqueous media have been systematically investigated. Experiments with individual components did not result in the production of gold nanoparticles except for the trans-cinnamaldehyde and linalool. The primary phytochemical, trans-cinnamaldehyde reduces $\mathrm{NaAuCl}_{4}$ in aqueous media to produce a purple-red solution of AuNPs and provide the required stability. The aldehyde itself is oxidized to cinnamic acid (as evidenced by ${ }^{1} \mathrm{H}$ NMR) which precipitated as a crystalline solid on the walls of the reaction vessel. To further evaluate the effective antioxidant power of trans-cinnamaldehyde present in cinnamon, the reduction potential values of different organic aldehydes from the literature have been carefully examined. The primary aromatic aldehydes (e.g. benzaldehyde) show reduction potential in the vicinity of $-1.5 \mathrm{~V}$, a value that is far below the reduction potential of $[\mathrm{AuCl} 4]^{-} / \mathrm{Au}(+0.99$ V).(42, 43) Considering these values, a redox couple of $\mathrm{NaAuCl}_{4}$-cinnamaldehyde is thermodynamically feasible and therefore cinnamaldehyde is able to reduce $\mathrm{NaAuCl}_{4}$ to 
gold nanoparticles. Linalool also reduced $\mathrm{NaAuCl}_{4}$ to generate AuNPs but they failed to stabilize nanoparticles in aqueous solution. The chemical constitutions of benzaldehyde, linalool, are comprised of alcoholic $-\mathrm{OH}$, aldehydic functional groups which may be responsible for the reduction of $\mathrm{NaAuCl}_{4}$. From the series of experiments performed using various phytochemicals present in cinnamon, it can be infer that only transcinnamaldehyde provides both reducing and stabilizing properties simultaneously during the nanoparticle formation.

The presence of carbohydrates for gold nanoparticle stabilization during the reduction process of $\mathrm{NaAuCl}_{4}$ by linalool in aqueous media (Fig. 2) has been further investigated. For carbohydrates, which are less soluble in aqueous media, the reactions were carried out by initially dissolving them at $\sim 70^{\circ} \mathrm{C}$, with subsequent addition of phytochemicals linalool to $\mathrm{NaAuCl}_{4}$ to produce gold nanoparticles. These results have clearly shown that coating of carbohydrates onto AuNPs provide good stability, whereas alcoholic or aldehydic components of cinnamon are directly involved in $\mathrm{NaAuCl}_{4}$ reduction. However, the cocktail effects of various phytochemicals present in cinnamon, including carbohydrates, linalool, trans-cinnamaldehyde provide dual reducing and stabilization properties simultaneously during the nanoparticle formation.

The assessment of in vitro stability and nontoxic features of newly fabricated nanomaterials is an important factor for biomedical applications. The in vitro stability of Cin-AuNPs has clearly shown that phytochemicals in cinnamon, when coated onto AuNPs, provide exceptional stability for extended periods of time in biologically relevant media. The addition of $10 \% \mathrm{NaCl}, 0.5 \%$ cysteine, $0.2 \mathrm{M}$ histidine, $0.5 \% \mathrm{HSA}$ and $0.5 \%$ 
BSA or 4-9 $\mathrm{pH}$ buffers to Cin-AuNPs solution did not cause any aggregation or decomposition of nanoparticles.

The cytotoxicity of Cin-AuNPs under in vitro conditions in human fibroblast cells was examined in terms of the effect of Cin-AuNPs on cell proliferation by MTT assay. After treatment with Cin-AuNPs for $24 \mathrm{~h}$, cells showed more than $80 \%$ viability at concentrations up to $1 \mathrm{mg} / \mathrm{mL}$ (Fig. 4). It has been reported that, a number of $\mathrm{Au}^{\mathrm{I}}$ and $\mathrm{Au}^{\mathrm{III}}$ complexes produce significant toxicity in cell culture media.(44) In contrast, cinnamon phytocemicals coated gold nanoparticles (Cin-AuNPs) showed minimal or no toxicity in primary human fibroblast cultures. This observation ensures that the phytochemicals present in cinnamon effectively reduce $\mathrm{NaAu}^{\mathrm{III}} \mathrm{Cl}_{4}$ in aqueous medium and provide the non-toxic phytochemicals surface coating onto AuNPs signifying biocompatible nature for in vivo administrations. The lack of any noticeable toxicity of Cin-AuNPs thus provides new opportunities for safe delivery and applications of such nanopahrmaceuticals in molecular imaging and therapy.

In order to explore the interaction of Cin-AuNPs with cancerous cells, we have investigated the effect of cellular internalization of Cin-AuNPs in prostate and breast cancer cells. Development of AuNPs with well defined smaller and narrow size distribution is of paramount importance for internalization of engineered nanoparticles within cellular matrix. Effective internalization of AuNPs within tumor cells offers excellent opportunities for biomedical imaging and therapy. It is widely accepted that the internalization of nanoparticles strongly depend on their physic-chemical characteristics including size, shape, and charge. DeSimone and co-workers have recently demonstrated that nanoparticles with either larger size or negative zeta potential exhibit no cellular 
internalizations.(45) To internalize negatively charged DNA molecules inside the cells, they utilized cationic ammonium ions as vectors which interact effectively with a negatively charged cell membrane, facilitating the charge-mediated endocytosis.(45) It is important to recognize that Cin-AuNPs also have a negative potential of $-31.0 \mathrm{mV}$. This means that Cin-AuNPs are expected to show minimal or no interaction with a negatively charged cell surface. However, significant internalization of Cin-AuNPs in PC 3 cells, as observed in our studies, strongly suggest that internalization of Cin-AuNPs does not happen through charge mediated endocytosis. The high propensity of Cin-AuNPs for endocytosis in PC-3 cells is presumably occurring through phagocytosis, involving the vesicular internalization of gold nanoparticles. It is important to note that the cellular membrane showed the formation of phagocytic cups (Fig. 5b), which are usually present in the cell wall if phagocytosis has occurred. In addition, AuNPs were found in endosomal vesciles as an individual probes in the form of dense mass of nanoparticles (Fig. 5). The localization of nanoparticles inside the vesicle supports the non-toxic nature of the Cin-AuNPs. Similar observations have been made by others attributing the nontoxic behavior of gold nanoparticles to the compartmentalization of nanoparticles in vesicles inside the cells.(46) The trapping of nanoparticles inside the vesicle is expected to prevent the interaction between cytoplasm and nucleus of the cell that resulted in a limited cytotoxicity.(46)

In order to demonstrate the importance of the synergistic effects of cinnamon phytochemicals toward the internalization of AuNPs in cancerous cells, the internalization behaviour of gold nanoparticles generated through cinnamaldehyde (one of the main components of cinnamon) were independently investigated in PC-3 cells. As 
shown in Fig. 5, gold nanoparticles produced by cinnamaldehyde showed significantly fewer AuNPs internalized in PC-3 cells as compared to Cin-AuNPs. These observations corroborate that the phytochemicals present in cinnamon when coated onto AuNPs provide optimal charge, hydrophobic pockets, and hydrodyanamic size-all of which singularly or synergistically result in excellent internalization of Cin-AuNPs in PC-3 and MCF-7 cells. In sharp contrast, cinnamaldehyde coated AuNPs fail to internalize in cancer cells as they presumably lack optimal charge, hydrophobic pockets, and hydrodyanamic sizes. This unique synergistic cocktail effect of Cin-AuNPs may provide new opportunities for generating biocompatible AuNPs for applications in in vitro and in vivo nanoparticulate-mediated imaging and therapy.

To further support the cell internalization of Cin-AuNPs, photoacoustic detection of PC-3 tumor cells incubated with Cin-AuNPs was performed. Photoacoustic technique is an attractive technique for potential applications in diagnosis of CTC. $(41,47)$ The sensitivity of photoacoustic imaging of tumor cells depends on their photon absorption characteristics. As the optical absorption is directly proportional to the number of nanoparticles embedded within cancer cells, it is important to develop gold nanoparticles with efficient propensity for selective internalization within tumor cells. Cin-AuNPs have shown extraordinary propensity for internalization within PC-3 cells (Fig. 5). This selective internalization of Cin-AuNPs within PC-3 cells of human origin provides threshold concentrations required for generating photoacoustic signals of individual nanoparticle embedded cancer cells (Fig 6). PA detection of PC-3 cells using Cin-AuNPs can provide a novel approach of utilizing phytochemical stabilized gold nanoparticles as optical contrast enhancement agents to detect CTC in cancer patients. 
The pharmacokinetics and organ accumulation ability of Cin-AuNPs were investigated by injecting the Cin-AuNPs via intravenous mode (IV) in normal mice models. The organ accumulation of AuNPs was monitored for a period of 24 hours post injection and the amount of gold in organs was estimated by atomic absorption spectroscopy (Fig. 7). In our earlier studies, it was established that maltose conjugated gold nanoparticles (MALT-AuNPs) predominantly accumulate in lungs because of collectin protein present in lung tissues.(7) Collectins are a group of surfactant proteins involved with innate immunity in the lungs.(48) Their role in immunity is to recognize and bind carbohydrate patterns to enhance phagocytosis and clearance of invading microorganisms.(48) It is possible that these collectins are recognizing the MALT sugar on the surface of the MALT-AuNPs and causing their accumulation in the lung tissue. Indeed, our present results clearly demonstrate that the biodistribution of Cin-AuNPs follow a similar trend. The primary objective of this biodistrubution studies were to investigate if Cin-AuNP's are stable in vivo. Uptake of nanoparticles in blood through serum proteins often limits the in vivo applications of gold nanoparticles. Our biodistribution data as shown in Figure 7 clearly demonstrates that Cin-AuNP's are cleared very efficiently through the blood stream and that there is minimal/no accumulation of gold nanoparticles in serum proteins. Excellent clearance of Cin-AuNPs from the blood suggests that cinnamon phytochemicals are bound to gold nanoparticles and rendering them robust in vivo stability. Therefore, Cin-AuNP's present realistic potential for applications in molecular imaging and therapy in vivo. Further in vivo studies on the in vivo applications of Cin-AuNP's are underway. 
Contrast-enhancement studies were carried out using phantoms prepared from Cin-AuNPs as tissue mimics. The higher mean density of gold compared to the background results in a contrast differential $\Delta \mathrm{HU}$ (HU=Hounsfield units). HU values were measured for increasing concentrations of Cin-AuNPs and results from each concentration are summarized in Fig. 9. A linear relationship is observed between CinAuNPs concentration and $\Delta \mathrm{HU}$. The slopes of both lines $(80$ and $140 \mathrm{kVp})$ are approximately 18.5 $\mathrm{HU}$ per milligram of gold per $\mathrm{mL}$ of volume in comparison to water. Quantitative analysis of CT values for each concentration reveals that the cinnamon phytochemicals coated AuNPs has consistent attenuation coefficients with increasing concentrations. This result clearly suggests that Cin-AuNPs may have potential for use in in vivo $\mathrm{CT}$ molecular imaging for the detection of cancerous lesions.

\section{CONCLUSION}

The redox systems of naturally available phytochemicals in cinnamon can be effectively used for generation of uniform sized gold nanoparticles with concomitant coating of phytochemicals. Therefore, cinnamon serves as a $100 \%$ green initiator for the synthesis and stabilization of gold nanoparticles derived from cinnamon phytochemicals. Cin-AuNPs are biocompatible, as established through MTT assays and cell internalization, without disturbing cellular machinery. The biodistribution results indicate that Cin-AuNPs were delivered effectively to lungs with minimal distribution to other organs and thereby can serve as lung imaging agent. Our in vitro studies also confirm the ability of Cin-AuNPs to serve as signal enhancers for the detection of individual cancer cells through photoacoustic technique. Furthermore, we showed through our preliminary 
X-ray CT experiments that the attenuation coefficient of the cinnamon stabilized gold nanoparticles increases with increasing concentrations of the matrix, thereby suggesting that this new generation of biocompatible Cin-AuNPs may be clinically useful as contrast enhancement molecular imaging agents for cancer diagnosis.

\section{ACKNOWLEDGEMENTS}

This work has been supported by grants from the National Institutes of Health/National Cancer Institute under the Cancer Nanotechnology Platform program (grant number: 5R01CA119412-01), NIH - 1R21CA128460-01; NIH-SBIR-Contract no. 241, and University of Missouri-Research Board - Program C8761 RB 06-030. We thank Prof. Henry W. White (University of Missouri, Columbia, MO USA) for his valuable comments and corrections of this manuscript. 


\section{REFERENCES}

1. M. D. Yang, Y. K. Liu, J. L. Shen, C. H. Wu, C. A. Lin, W. H. Chang, H. H. Wang, H. I. Yeh, W. H. Chan, and W. J. Parak. Improvement of conversion efficiency for multi-junction solar cells by incorporation of Au nanoclusters. Opt Express 16: 1575415758 (2008).

2. P. K. Jain, X. Huang, I. H. El-Sayed and M. A. El-Sayed. Noble metals on the nanoscale: optical and photothermal properties and some applications in imaging, sensing, biology, and medicine. Acc Chem Res. 41: 1578-1586 (2008).

3. C. J. Murphy, A. M. Gole, J. W. Stone, P. N. Sisco, A. M. Alkilany, E. C. Goldsmith and S. C. Baxter. Gold nanoparticles in biology: beyond toxicity to cellular imaging. Acc Chem Res. 41: 1721-1730 (2008).

4. Y. Wang, C. A. Mirkin and S. J. Park. Nanofabrication beyond electronics. ACS Nano 3: 1049-1056 (2009).

5. K. Esumi, A. Kameo, A. Suzuki, and K. Torigoe. Preparation of gold nanoparticles in formamide and N,N-dimethylformamide in the presence of poly(amidoamine) dendrimers with surface methyl ester groups. Colloids and Surfaces, A: Physicochemical and Engineering Aspects 189: 155-161 (2001).

6. A. Feitz, J, Guan, D. Waite. Process for producing a nanoscale zero-valent metal. US Patent Application Publication. US2006/0083924 A1 (2006).

7. G. M. Fent, S. W. Casteel, D. Y. Kim, R. Kannan, K. Katti and N. Chanda, Biodistribution of maltose and gum arabic hybrid gold nanoparticles after intravenous injection in juvenile swine. Nanomedicine:NBM 5: 128-135 (2009).

8. K. Katti, N. Chanda, R, Shukla, A. Zambre, T. Suibramanian, R. R. Kulkarni, R, Kannan, and K. V. Katti. Green Nanotechnology from Cumin Phytochemicals: Generation of Biocompatible Gold Nanoparticles. International Journal of Nanotechnology: Biomedicine 1: B39-B52 (2009).

9. K. K. Katti, V. Kattamuri, S. Bhaskaran, R. Kannan, and K. V. Katti. Facile and General Method for Synthesis of Sugar-Coated Gold Nanoparticles. International Journal of Nanotechnology: Biomedicine. 1: B53-B59 (2009).

10. V. Kattumuri, K. Katti, S. Bhaskaran, E. J. Boote, S. W. Casteel, G. M. Fent, D. J. Robertson, M. Chandrasekhar, R. Kannan and K. V. Katti. Gum arabic as a phytochemical construct for the stabilization of gold nanoparticles: in vivo pharmacokinetics and X-ray-contrast-imaging studies. Small 3: 333-341 (2007).

11. V. Kattumuri, M. Chandrasekhar, S. Guha, R. Kannan, K. V. Katti, T. K. Ghosh, and R. J. Patel. Agarose-stabilized gold nanoparticles for surface-enhanced Raman spectroscopic detection of DNA nucleosides. Appl. Phys. Lett. 88: 153114 (2006).

12. S. Nune, N. Chanda, R, Shukla, K. Katti, R. R. Kulkarni, S. Thilakavathy, S. Mekapothula, R. Kannan, and K. V. Katti. Green nanotechnology from tea: phytochemicals in tea as building blocks for production of biocompatible gold nanoparticles. J. Mater. Chem. 19: 2912-2920 (2009).

13. R. Shukla, S. K. Nune, N. Chanda, K. Katti, S. Mekapothula, R. R. Kulkarni, W. V. Welshons, R. Kannan, and K. V. Katti. Soybeans as a phytochemical reservoir for the production and stabilization of biocompatible gold nanoparticles. Small 4: 1425-1436 (2008).

14. R. Shukla, S. K. Nune, N. Chanda, K. Katti, S. Mekapothula, R. R. Kulkarni, W. V. Welshons, R. Kannan, and K. V. Katti. Soybeans as a phytochemical reservoir for the 
production and stabilization of biocompatible gold nanoparticles. Science Editors' Choice 322: 167 (2008).

15. N. Hoyoku, S. Yoshiko, T, Harukuni, M. Mitsuharu. Cancer Control by Phytochemicals. Current Pharmaceutical Design. 13: 3394-3399 (2007).

16. I. T. Johnson, Phytochemicals and Cancer. Proceedings of the Nutrition Society. 66: 207-215 (2007).

17. M. Dekker, R. Verkrek. Dealing with variability in food production chains: a tool to enhance the sensitivity of epidemiological studies on phytochemicals. Eur J Nutr. 42: 67-72 (2003).

18. B. Holst, G. Williamson. Nutrients and phytochemicals: from bioavailability to bioefficacy beyond antioxidants. Curr Opin Biotechnol. 19: 73-82 (2008).

19. R. T. Gow, D. Li, G. W. Sypert, and R. S. Alberte. Extracts and Methods Comprising Cinnamon Species. US Patent Application Publication US 2007/0292540 Al (2007).

20. S. Mathew, and T. E. Abraham. Studies on the antioxidant activities of cinnamon (Cinnamomum verum) bark extracts, through various in vitro models. Food Chemistry 94: 520-528 (2006).

21. P. Lopez, C. Sanchez, R. Batlle and C. Nerin. Vapor-phase activities of cinnamon, thyme, and oregano essential oils and key constituents against foodborne microorganisms. J Agric Food Chem. 55: 4348-4356 (2007).

22. B. Shan, Y. Z. Cai, J. D. Brooks, and H. Corke, Antibacterial properties and major bioactive components of cinnamon stick (Cinnamomum burmannii): activity against foodborne pathogenic bacteria. J Agric Food Chem. 55: 5484-5490 (2007).

23. B. Shan, Y. Z. Cai, M. Sun, and H. Corke, Antioxidant capacity of 26 spice extracts and characterization of their phenolic constituents. J Agric Food Chem. 53: 7749-7759 (2005).

24. J. Usta, S. Kreydiyyeh, P. Barnabe, Y. Bou-Moughlabay, and H. NakkashChmaisse. Comparative study on the effect of cinnamon and clove extracts and their main components on different types of ATPases. Hum Exp Toxicol. 22: 355-362 (2003).

25. H. Schmidt-Kloiber, and G. Paltauf. Measuring optical tissue data using pulsed photoacoustic spectroscopy (PPAS). Biomed Tech. (Berl) 42 Suppl: 227-228 (1997).

26. O. R. Faha, OSIRIX: An Oepn Source Platform for Advanced Multimodality Medical Imaging. IEEE Information \& Communications Technology (2006).

27. N. Chanda, R. Shukla, K. V. Katti, and R. Kannan. Gastrin Releasing Protein Receptor -Specific Gold Nanorods: Breast and Prostate Tumor-avid Nanovectors for Molecular Imaging. Nano Letter 9: 1798-1805 (2009).

28. R. Kannan, V. Rahing, C. Cutler, R. Pandrapragada, K. K. Katti, V. Kattumuri, J. D. Robertson, S. J. Casteel, S. Jurisson, C. Smith, E. Boote, and K. V. Katti. Nanocompatible chemistry toward fabrication of target-specific gold nanoparticles. J Am Chem Soc. 128: 11342-11343 (2006).

29. R. Kannan, C. Cutler, V. Rahing, C. Smith, and K. Katti. Bioconjugated radioactive gold nanoparticles and their in vivo targeting abilities in small animal models. J Nucl Med 47 (Supplement 1), 510 (2006).

30. R. Kannan, K. V. Katti, K. K. Katti, H. W. White, and C. S. Cutler. Methods and articles for gold nanoparticle production. US 2007/0051202 A1, US Patent Application Publication (2007). 
31. K. Katti, R. Kannan, K. K. Katti, S. Bhaskaran, and R. K. Pandrapragada. Optimization and Production of Gold and Silver Nanoparticles for Potential Imaging Applications. Molecular imaging 3: 278 (2004).

32. N. Chanda, P. Kan, L. D. Watkinson, R. Shukla, A. Zambre, T. L. Carmack, H. Engelbrecht, J. R. Lever, K. K. Katti, G. M. Fent, S. W. Casteel, C. J. Smith, W. H. Miller, S. Jurisson, E. Boote, J. D. Robertson, C. Cutler, M. Dobrovolskaia, R. Kannan, and K. V. Katti. Radioactive gold nanoparticles in cancer therapy: Therapeutic efficacy studies of ${ }^{198} \mathrm{AuNP}-\mathrm{GA}$ nanoconstruct in prostate tumor bearing mice. Nanomedicine: NBM 6: 201-209 (2009).

33. N. Chanda, V. Kattumuri, R. Shukla, A. Zambre, K. Katti, A. Upendran, R. R. Kulkarni, P. Kan, G. M. Fent, S. W. Casteel, C. J. Smith, E. Boote, J. D. Robertson, C. Cutler, J. Lever, K. V. Katti, R. Kannan. Bombesin Functionalized Gold Nanoparticles Show In vitro and In vivo Cancer Receptor Specificity. Proc. Natl. Acad. Sci. USA 107: 8760-5 (2010).

34. H-K. Na, and Y. J. Surh. Intracellular signaling network as a prime chemopreventive target of (-)-epigallocatechin gallate. Mol Nutr Food Res. 50: 152-159 (2006).

35. J. Sun de, Y. Liu, D. C. Lu, W. Kim, J. H. Lee, J. Maynard, and A. Deisseroth. Endothelin-3 growth factor levels decreased in cervical cancer compared with normal cervical epithelial cells. Hum Pathol. 38: 1047-1056 (2007).

36. M. T. Baratta, H. J. D. Dorman, S. G. Deans, A. C. Figueiredo, J. G. Barroso, and G. Ruberto. Antimicrobial and antioxidant properties of some commercial essential oils. Flavour Fragr. J. 13: 235-244 (1998).

37. S. Mallidi, P. P. Joshi, K. Sokolov and S. Emelianov. On sensitivity of molecular specific photoacoustic imaging using plasmonic gold nanoparticles. Conf Proc IEEE Eng Med Biol Soc. 6338-6340 (2009).

38. S. Mallidi, T. Larson, J. Tam, P. P. Joshi, A. Karpiouk, K. Sokolov, and S. Emelianov. Multiwavelength photoacoustic imaging and plasmon resonance coupling of gold nanoparticles for selective detection of cancer. Nano Lett. 9: 2825-2831 (2009).

39. D. Pan, M. Pramanik, A. Senpan, S. Ghosh, S. A. Wickline, L. V. Wang, and G. M. Lanza. Near infrared photoacoustic detection of sentinel lymph nodes with gold nanobeacons. Biomaterials 31: 4088-4093 (2010).

40. D. Pan, M. Pramanik, A. Senpan, X. Yang, K. H. Song, M. J. Scott, H. Zhang, P. J. Gaffney, S. A. Wickline, et al., Molecular photoacoustic tomography with colloidal nanobeacons. Angew Chem Int Ed. 48: 4170-4173 (2009).

41. J. Viator, S. Gupta, B. S. Goldschmidt, K. Bhattacharyya, R. Kannan, R. Shukla, P. S. Dale, E. Boote, K. V. Katti. Detection of Gold Nanoparticle Enhanced Prostate Cancer Cells Using Photoacoustic Flowmetry with Optical Reflectance. Journal of Biomedical Nanotechnology 6: 1-5 (2010).

42. N. R. Armstrong, R. K. Quinn, and N. E. Vanderborgh. Voltammetry in sulfolane. Electrochemical behavior of benzaldehyde and substituted benzaldehydes. Analytical Chemistry 46: 1759-1764 (1974).

43. L. Au, X. Lu, and Y. Xia. A Comparative Study of Galvanic Replacement Reactions Involving Ag Nanocubes and $\mathrm{AuCl}(2)$ or $\mathrm{AuCl}(4)$. Adv Mater Deerfield 20: 2517-2522 (2008).

44. I. C. Shaw. Gold-based therapeutic agents. Chem Rev. 99: 2589-2600 (1999). 
45. S. E. Gratton, P. A. Ropp, P. D. Pohlhaus, J. C. Luft, V. J. Madden, M. E. Napier, and J. M. DeSimone. The effect of particle design on cellular internalization pathways. Proc Natl Acad Sci U S A 105: 11613-11618 (2008).

46. T. S. Hauck, A. A. Ghazani, and W. C. Chan. Assessing the effect of surface chemistry on gold nanorod uptake, toxicity, and gene expression in mammalian cells. Small 4: 153-159 (2008).

47. E. I. Galanzha, E. V. Shashkov, P. M. Spring, J. Y. Suen, and V. P. Zharov. In vivo, noninvasive, label-free detection and eradication of circulating metastatic melanoma cells using two-color photoacoustic flow cytometry with a diode laser. Cancer Res. 69: 7926-7934 (2009).

48. T. P. Hickling, H. Clark, R. Malhotra, and R. B. Sim. Collectins and their role in lung immunity. J Leukoc Biol. 75: 27-33 (2004). 